\title{
Partial Differentiation of Real Binary Functions
}

\author{
Bing Xie \\ Qingdao University of Science \\ and Technology \\ China
}

\author{
Xiquan Liang \\ Qingdao University of Science \\ and Technology \\ China
}

\author{
Hongwei Li \\ Qingdao University of Science \\ and Technology \\ China
}

\begin{abstract}
Summary. In this article, we define two single-variable functions SVF1 and SVF2, then discuss partial differentiation of real binary functions by dint of one variable function SVF1 and SVF2. The main properties of partial differentiation are shown [7].
\end{abstract}

MML identifier: $\underline{\text { PDIFF_2 }}$, version: $\underline{7.9 .03 \quad 4.104 .1021}$

The articles [14], [4], [15], [5], [1], [8], [10], [9], [2], [3], [13], [6], [12], [11], and [7] provide the notation and terminology for this paper.

\section{Preliminaries}

For simplicity, we adopt the following convention: $x, x_{0}, y, y_{0}, r$ are real numbers, $z, z_{0}$ are elements of $\mathcal{R}^{2}, Z$ is a subset of $\mathcal{R}^{2}, f, f_{1}, f_{2}$ are partial functions from $\mathcal{R}^{2}$ to $\mathbb{R}, R$ is a rest, and $L$ is a linear function.

Next we state two propositions:

(1) $\operatorname{dom} \operatorname{proj}(1,2)=\mathcal{R}^{2}$ and $\operatorname{rng} \operatorname{proj}(1,2)=\mathbb{R}$ and for all elements $x, y$ of $\mathbb{R}$ holds $(\operatorname{proj}(1,2))(\langle x, y\rangle)=x$.

(2) $\operatorname{dom} \operatorname{proj}(2,2)=\mathcal{R}^{2}$ and $\operatorname{rng} \operatorname{proj}(2,2)=\mathbb{R}$ and for all elements $x, y$ of $\mathbb{R}$ holds $(\operatorname{proj}(2,2))(\langle x, y\rangle)=y$. 


\section{Partial Differentiation of Real Binary Functions}

Let $f$ be a partial function from $\mathcal{R}^{2}$ to $\mathbb{R}$ and let $z$ be an element of $\mathcal{R}^{2}$. The functor $\operatorname{SVF} 1(f, z)$ yielding a partial function from $\mathbb{R}$ to $\mathbb{R}$ is defined by:

(Def. 1) $\operatorname{SVF} 1(f, z)=f \cdot \operatorname{reproj}(1, z)$.

The functor $\operatorname{SVF} 2(f, z)$ yields a partial function from $\mathbb{R}$ to $\mathbb{R}$ and is defined as follows:

(Def. 2) $\operatorname{SVF} 2(f, z)=f \cdot \operatorname{reproj}(2, z)$.

Next we state two propositions:

(3) If $z=\langle x, y\rangle$ and $f$ is partially differentiable in $z$ w.r.t. 1 coordinate, then $\operatorname{SVF} 1(f, z)$ is differentiable in $x$.

(4) If $z=\langle x, y\rangle$ and $f$ is partially differentiable in $z$ w.r.t. 2 coordinate, then $\operatorname{SVF} 2(f, z)$ is differentiable in $y$.

Let $f$ be a partial function from $\mathcal{R}^{2}$ to $\mathbb{R}$ and let $z$ be an element of $\mathcal{R}^{2}$. We say that $f$ is partial differentiable on 1st coordinate in $z$ if and only if:

(Def. 3) There exist real numbers $x_{0}, y_{0}$ such that $z=\left\langle x_{0}, y_{0}\right\rangle$ and $\operatorname{SVF} 1(f, z)$ is differentiable in $x_{0}$.

We say that $f$ is partial differentiable on 2 nd coordinate in $z$ if and only if:

(Def. 4) There exist real numbers $x_{0}, y_{0}$ such that $z=\left\langle x_{0}, y_{0}\right\rangle$ and $\operatorname{SVF} 2(f, z)$ is differentiable in $y_{0}$.

Next we state two propositions:

(5) Suppose $z=\left\langle x_{0}, y_{0}\right\rangle$ and $f$ is partial differentiable on 1st coordinate in $z$. Then there exists a neighbourhood $N$ of $x_{0}$ such that $N \subseteq \operatorname{dom} \operatorname{SVF} 1(f, z)$ and there exist $L, R$ such that for every $x$ such that $x \in N$ holds $(\operatorname{SVF} 1(f, z))(x)-(\operatorname{SVF} 1(f, z))\left(x_{0}\right)=L\left(x-x_{0}\right)+R\left(x-x_{0}\right)$.

(6) Suppose $z=\left\langle x_{0}, y_{0}\right\rangle$ and $f$ is partial differentiable on 2nd coordinate in $z$. Then there exists a neighbourhood $N$ of $y_{0}$ such that $N \subseteq$ dom SVF2 $(f, z)$ and there exist $L, R$ such that for every $y$ such that $y \in N$ holds $(\operatorname{SVF} 2(f, z))(y)-(\operatorname{SVF} 2(f, z))\left(y_{0}\right)=L\left(y-y_{0}\right)+R\left(y-y_{0}\right)$.

Let $f$ be a partial function from $\mathcal{R}^{2}$ to $\mathbb{R}$ and let $z$ be an element of $\mathcal{R}^{2}$. Let us observe that $f$ is partial differentiable on 1st coordinate in $z$ if and only if the condition (Def. 5) is satisfied.

(Def. 5) There exist real numbers $x_{0}, y_{0}$ such that

(i) $z=\left\langle x_{0}, y_{0}\right\rangle$, and

(ii) there exists a neighbourhood $N$ of $x_{0}$ such that $N \subseteq \operatorname{dom} \operatorname{SVF} 1(f, z)$ and there exist $L, R$ such that for every $x$ such that $x \in N$ holds $(\operatorname{SVF} 1(f, z))(x)-(\operatorname{SVF} 1(f, z))\left(x_{0}\right)=L\left(x-x_{0}\right)+R\left(x-x_{0}\right)$.

Let $f$ be a partial function from $\mathcal{R}^{2}$ to $\mathbb{R}$ and let $z$ be an element of $\mathcal{R}^{2}$. Let us observe that $f$ is partial differentiable on 2nd coordinate in $z$ if and only if the condition (Def. 6) is satisfied. 
(Def. 6) There exist real numbers $x_{0}, y_{0}$ such that

(i) $z=\left\langle x_{0}, y_{0}\right\rangle$, and

(ii) there exists a neighbourhood $N$ of $y_{0}$ such that $N \subseteq \operatorname{domSVF} 2(f, z)$ and there exist $L, R$ such that for every $y$ such that $y \in N$ holds $(\operatorname{SVF} 2(f, z))(y)-(\operatorname{SVF} 2(f, z))\left(y_{0}\right)=L\left(y-y_{0}\right)+R\left(y-y_{0}\right)$.

Next we state two propositions:

(7) Let $f$ be a partial function from $\mathcal{R}^{2}$ to $\mathbb{R}$ and $z$ be an element of $\mathcal{R}^{2}$. Then $f$ is partial differentiable on 1st coordinate in $z$ if and only if $f$ is partially differentiable in $z$ w.r.t. 1 coordinate.

(8) Let $f$ be a partial function from $\mathcal{R}^{2}$ to $\mathbb{R}$ and $z$ be an element of $\mathcal{R}^{2}$. Then $f$ is partial differentiable on 2nd coordinate in $z$ if and only if $f$ is partially differentiable in $z$ w.r.t. 2 coordinate.

Let $f$ be a partial function from $\mathcal{R}^{2}$ to $\mathbb{R}$ and let $z$ be an element of $\mathcal{R}^{2}$. The functor partdiff $1(f, z)$ yielding a real number is defined by:

(Def. 7) $\operatorname{partdiff1}(f, z)=\operatorname{partdiff}(f, z, 1)$.

The functor partdiff2 $(f, z)$ yielding a real number is defined as follows:

(Def. 8) $\operatorname{partdiff} 2(f, z)=\operatorname{partdiff}(f, z, 2)$.

One can prove the following propositions:

(9) Suppose $z=\left\langle x_{0}, y_{0}\right\rangle$ and $f$ is partial differentiable on 1st coordinate in $z$. Then $r=\operatorname{partdiff} 1(f, z)$ if and only if there exist real numbers $x_{0}$, $y_{0}$ such that $z=\left\langle x_{0}, y_{0}\right\rangle$ and there exists a neighbourhood $N$ of $x_{0}$ such that $N \subseteq \operatorname{dom} \operatorname{SVF} 1(f, z)$ and there exist $L, R$ such that $r=L(1)$ and for every $x$ such that $x \in N$ holds $(\operatorname{SVF} 1(f, z))(x)-(\operatorname{SVF} 1(f, z))\left(x_{0}\right)=$ $L\left(x-x_{0}\right)+R\left(x-x_{0}\right)$.

(10) Suppose $z=\left\langle x_{0}, y_{0}\right\rangle$ and $f$ is partial differentiable on 2nd coordinate in $z$. Then $r=\operatorname{partdiff2}(f, z)$ if and only if there exist real numbers $x_{0}$, $y_{0}$ such that $z=\left\langle x_{0}, y_{0}\right\rangle$ and there exists a neighbourhood $N$ of $y_{0}$ such that $N \subseteq \operatorname{domSVF} 2(f, z)$ and there exist $L, R$ such that $r=L(1)$ and for every $y$ such that $y \in N$ holds $(\operatorname{SVF} 2(f, z))(y)-(\operatorname{SVF} 2(f, z))\left(y_{0}\right)=$ $L\left(y-y_{0}\right)+R\left(y-y_{0}\right)$.

(11) If $z=\left\langle x_{0}, y_{0}\right\rangle$ and $f$ is partial differentiable on 1st coordinate in $z$, then $\operatorname{partdiff} 1(f, z)=(\operatorname{SVF} 1(f, z))^{\prime}\left(x_{0}\right)$.

(12) If $z=\left\langle x_{0}, y_{0}\right\rangle$ and $f$ is partial differentiable on 2nd coordinate in $z$, then $\operatorname{partdiff} 2(f, z)=(\operatorname{SVF} 2(f, z))^{\prime}\left(y_{0}\right)$.

Let $f$ be a partial function from $\mathcal{R}^{2}$ to $\mathbb{R}$ and let $Z$ be a set. We say that $f$ is partial differentiable w.r.t. 1st coordinate on $Z$ if and only if:

(Def. 9) $Z \subseteq \operatorname{dom} f$ and for every element $z$ of $\mathcal{R}^{2}$ such that $z \in Z$ holds $f\lceil Z$ is partial differentiable on 1st coordinate in $z$.

We say that $f$ is partial differentiable w.r.t. 2 nd coordinate on $Z$ if and only if: 
(Def. 10) $Z \subseteq \operatorname{dom} f$ and for every element $z$ of $\mathcal{R}^{2}$ such that $z \in Z$ holds $f\lceil Z$ is partial differentiable on 2 nd coordinate in $z$.

One can prove the following two propositions:

(13) Suppose $f$ is partial differentiable w.r.t. 1st coordinate on $Z$. Then $Z \subseteq$ $\operatorname{dom} f$ and for every $z$ such that $z \in Z$ holds $f$ is partial differentiable on 1st coordinate in $z$.

(14) Suppose $f$ is partial differentiable w.r.t. 2nd coordinate on $Z$. Then $Z \subseteq \operatorname{dom} f$ and for every $z$ such that $z \in Z$ holds $f$ is partial differentiable on 2 nd coordinate in $z$.

Let $f$ be a partial function from $\mathcal{R}^{2}$ to $\mathbb{R}$ and let $Z$ be a set. Let us assume that $f$ is partial differentiable w.r.t. 1st coordinate on $Z$. The functor $f_{\uparrow Z}^{1 \text { st }}$ yielding a partial function from $\mathcal{R}^{2}$ to $\mathbb{R}$ is defined as follows:

(Def. 11) $\operatorname{dom}\left(f_{\lceil Z}^{1 \mathrm{st}}\right)=Z$ and for every element $z$ of $\mathcal{R}^{2}$ such that $z \in Z$ holds $f_{\lceil Z}^{1 \text { st }}(z)=\operatorname{partdiff} 1(f, z)$.

Let $f$ be a partial function from $\mathcal{R}^{2}$ to $\mathbb{R}$ and let $Z$ be a set. Let us assume that $f$ is partial differentiable w.r.t. 2 nd coordinate on $Z$. The functor $f_{\Gamma Z}^{2 \text { nd }}$ yielding a partial function from $\mathcal{R}^{2}$ to $\mathbb{R}$ is defined as follows:

(Def. 12) $\operatorname{dom}\left(f_{\Gamma Z}^{2 \text { nd }}\right)=Z$ and for every element $z$ of $\mathcal{R}^{2}$ such that $z \in Z$ holds $f_{\uparrow Z}^{2 \mathrm{nd}}(z)=\operatorname{partdiff} 2(f, z)$.

\section{Main Properties of Partial Differentiation of Real Binary FUNCTIONS}

We now state a number of propositions:

(15) Let $z_{0}$ be an element of $\mathcal{R}^{2}$ and $N$ be a neighbourhood of $(\operatorname{proj}(1,2))\left(z_{0}\right)$. Suppose $f$ is partial differentiable on 1st coordinate in $z_{0}$ and $N \subseteq$ dom $\operatorname{SVF} 1\left(f, z_{0}\right)$. Let $h$ be a convergent to 0 sequence of real numbers and $c$ be a constant sequence of real numbers. Suppose $\operatorname{rng} c=\left\{(\operatorname{proj}(1,2))\left(z_{0}\right)\right\}$ and $\operatorname{rng}(h+c) \subseteq N$. Then $h^{-1}\left(\operatorname{SVF} 1\left(f, z_{0}\right) \cdot(h+c)-\operatorname{SVF} 1\left(f, z_{0}\right) \cdot c\right)$ is convergent and partdiff1 $\left(f, z_{0}\right)=\lim \left(h^{-1}\left(\operatorname{SVF} 1\left(f, z_{0}\right) \cdot(h+c)-\operatorname{SVF} 1\left(f, z_{0}\right)\right.\right.$. c)).

(16) Let $z_{0}$ be an element of $\mathcal{R}^{2}$ and $N$ be a neighbourhood of $(\operatorname{proj}(2,2))\left(z_{0}\right)$. Suppose $f$ is partial differentiable on 2 nd coordinate in $z_{0}$ and $N \subseteq$ dom SVF2 $\left(f, z_{0}\right)$. Let $h$ be a convergent to 0 sequence of real numbers and $c$ be a constant sequence of real numbers. Suppose $\operatorname{rng} c=\left\{(\operatorname{proj}(2,2))\left(z_{0}\right)\right\}$ and $\operatorname{rng}(h+c) \subseteq N$. Then $h^{-1}\left(\operatorname{SVF} 2\left(f, z_{0}\right) \cdot(h+c)-\operatorname{SVF} 2\left(f, z_{0}\right) \cdot c\right)$ is convergent and partdiff2 $\left(f, z_{0}\right)=\lim \left(h^{-1}\left(\operatorname{SVF} 2\left(f, z_{0}\right) \cdot(h+c)-\operatorname{SVF} 2\left(f, z_{0}\right)\right.\right.$. c)).

(17) Suppose $f_{1}$ is partial differentiable on 1st coordinate in $z_{0}$ and $f_{2}$ is partial differentiable on 1 st coordinate in $z_{0}$. Then $f_{1}+f_{2}$ is par- 
tial differentiable on 1 st coordinate in $z_{0}$ and partdiff $1\left(f_{1}+f_{2}, z_{0}\right)=$ $\operatorname{partdiff} 1\left(f_{1}, z_{0}\right)+\operatorname{partdiff} 1\left(f_{2}, z_{0}\right)$.

(18) Suppose $f_{1}$ is partial differentiable on 2 nd coordinate in $z_{0}$ and $f_{2}$ is partial differentiable on 2 nd coordinate in $z_{0}$. Then $f_{1}+f_{2}$ is partial differentiable on 2 nd coordinate in $z_{0}$ and partdiff $2\left(f_{1}+f_{2}, z_{0}\right)=$ $\operatorname{partdiff} 2\left(f_{1}, z_{0}\right)+\operatorname{partdiff} 2\left(f_{2}, z_{0}\right)$.

(19) Suppose $f_{1}$ is partial differentiable on 1 st coordinate in $z_{0}$ and $f_{2}$ is partial differentiable on 1 st coordinate in $z_{0}$. Then $f_{1}-f_{2}$ is partial differentiable on 1 st coordinate in $z_{0}$ and partdiff1 $\left(f_{1}-f_{2}, z_{0}\right)=$ $\operatorname{partdiff} 1\left(f_{1}, z_{0}\right)-\operatorname{partdiff} 1\left(f_{2}, z_{0}\right)$.

(20) Suppose $f_{1}$ is partial differentiable on 2 nd coordinate in $z_{0}$ and $f_{2}$ is partial differentiable on 2 nd coordinate in $z_{0}$. Then $f_{1}-f_{2}$ is partial differentiable on 2 nd coordinate in $z_{0}$ and partdiff $2\left(f_{1}-f_{2}, z_{0}\right)=$ $\operatorname{partdiff} 2\left(f_{1}, z_{0}\right)-\operatorname{partdiff} 2\left(f_{2}, z_{0}\right)$.

(21) Suppose $f$ is partial differentiable on 1st coordinate in $z_{0}$. Then $r f$ is partial differentiable on 1 st coordinate in $z_{0}$ and $\operatorname{partdiff} 1\left(r f, z_{0}\right)=$ $r \cdot \operatorname{partdiff} 1\left(f, z_{0}\right)$.

(22) Suppose $f$ is partial differentiable on 2 nd coordinate in $z_{0}$. Then $r f$ is partial differentiable on 2 nd coordinate in $z_{0}$ and partdiff $2\left(r f, z_{0}\right)=$ $r \cdot \operatorname{partdiff} 2\left(f, z_{0}\right)$.

(23) Suppose $f_{1}$ is partial differentiable on 1st coordinate in $z_{0}$ and $f_{2}$ is partial differentiable on 1 st coordinate in $z_{0}$. Then $f_{1} f_{2}$ is partial differentiable on 1st coordinate in $z_{0}$.

(24) Suppose $f_{1}$ is partial differentiable on 2nd coordinate in $z_{0}$ and $f_{2}$ is partial differentiable on 2 nd coordinate in $z_{0}$. Then $f_{1} f_{2}$ is partial differentiable on 2 nd coordinate in $z_{0}$.

(25) Let $z_{0}$ be an element of $\mathcal{R}^{2}$. Suppose $f$ is partial differentiable on 1st coordinate in $z_{0}$. Then $\operatorname{SVF} 1\left(f, z_{0}\right)$ is continuous in $(\operatorname{proj}(1,2))\left(z_{0}\right)$.

(26) Let $z_{0}$ be an element of $\mathcal{R}^{2}$. Suppose $f$ is partial differentiable on 2 nd coordinate in $z_{0}$. Then $\operatorname{SVF} 2\left(f, z_{0}\right)$ is continuous in $(\operatorname{proj}(2,2))\left(z_{0}\right)$.

(27) If $f$ is partial differentiable on 1st coordinate in $z_{0}$, then there exists $R$ such that $R(0)=0$ and $R$ is continuous in 0 .

(28) If $f$ is partial differentiable on 2 nd coordinate in $z_{0}$, then there exists $R$ such that $R(0)=0$ and $R$ is continuous in 0 .

\section{REFERENCES}

[1] Grzegorz Bancerek. The ordinal numbers. Formalized Mathematics, 1(1):91-96, 1990.

[2] Grzegorz Bancerek and Krzysztof Hryniewiecki. Segments of natural numbers and finite sequences. Formalized Mathematics, 1(1):107-114, 1990.

[3] Czesław Byliński. Finite sequences and tuples of elements of a non-empty sets. Formalized Mathematics, 1(3):529-536, 1990. 
[4] Czesław Byliński. Functions and their basic properties. Formalized Mathematics, 1(1):5565, 1990.

[5] Czesław Byliński. Partial functions. Formalized Mathematics, 1(2):357-367, 1990.

[6] Agata Darmochwał. The Euclidean space. Formalized Mathematics, 2(4):599-603, 1991.

[7] Noboru Endou, Yasunari Shidama, and Keiichi Miyajima. Partial differentiation on normed linear spaces $\nabla^{n}$. Formalized Mathematics, 15(2):65-72, 2007.

[8] Krzysztof Hryniewiecki. Basic properties of real numbers. Formalized Mathematics, $1(\mathbf{1}): 35-40,1990$.

[9] Jarosław Kotowicz. Convergent sequences and the limit of sequences. Formalized Mathematics, 1(2):273-275, 1990.

[10] Jarosław Kotowicz. Real sequences and basic operations on them. Formalized Mathematics, 1(2):269-272, 1990.

[11] Konrad Raczkowski and Paweł Sadowski. Real function continuity. Formalized Mathematics, 1(4):787-791, 1990.

[12] Konrad Raczkowski and Paweł Sadowski. Real function differentiability. Formalized Mathematics, 1(4):797-801, 1990.

[13] Konrad Raczkowski and Paweł Sadowski. Topological properties of subsets in real numbers. Formalized Mathematics, 1(4):777-780, 1990.

[14] Zinaida Trybulec. Properties of subsets. Formalized Mathematics, 1(1):67-71, 1990.

[15] Edmund Woronowicz. Relations defined on sets. Formalized Mathematics, 1(1):181-186, 1990. 